\title{
Quasiparton distribution functions at NNLO: Flavor nondiagonal quark contributions
}

\author{
Long-Bin Chen, ${ }^{1}$ Wei Wang, ${ }^{2, *}$ and Ruilin Zhu $\odot^{3,4, \dagger}$ \\ ${ }^{1}$ School of Physics and Materials Science, Guangzhou University, Guangzhou 510006, China \\ ${ }^{2} I N P A C$, SKLPPC, MOE KLPPC, School of Physics and Astronomy, Shanghai Jiao Tong University, \\ Shanghai 200240, China \\ ${ }^{3}$ Department of Physics and Institute of Theoretical Physics, Nanjing Normal University, \\ Nanjing, Jiangsu 210023, China \\ ${ }^{4}$ Nuclear Science Division, Lawrence Berkeley National Laboratory, Berkeley, California 94720, USA
}

(Received 29 May 2020; accepted 13 July 2020; published 27 July 2020)

\begin{abstract}
We present a next-to-next-to-leading order (NNLO) calculation of the quasiparton distribution functions (quasi-PDFs) in the large momentum effective theory (LaMET). We focus on the flavor nondiagonal quarkquark channel and demonstrate the LaMET factorization at the NNLO accuracy in the modified minimal subtraction scheme. The matching coefficient between the quasi-PDF and the light-cone PDF is derived. This provides a first step towards a complete NNLO analysis of quasi-PDFs and to better understand the nucleon structures from the first principle of QCD.
\end{abstract}

DOI: 10.1103/PhysRevD.102.011503

\section{INTRODUCTION}

Understanding the underlying structure of nucleons from degrees of quarks and gluons has been a long-standing goal in hadron physics. Since deep-inelastic scattering experiments at Stanford Linear Accelerator Center in the late 1960s, the proton structure has been explored in various hard scattering processes [1]. The key results involve the parton distribution functions (PDFs), defined as momentum distributions of quarks and gluons in an infinite-momentum hadron. These distribution functions are normally referred as the light-cone PDFs or the collinear PDFs. In high energy experiments at the lepton-hadron and hadron-hadron colliders, the PDFs are also the important ingredients to characterize the structure of hadrons and make predictions for various processes to test the standard model and probe the new physics beyond. Though the scale evolution of PDFs beyond leading order (LO) into next-to-next-to-next-to leading order (NNNLO) have been performed in literature [2-6], calculating the PDFs and more generally light-cone observables from first principle of quantum chromodynamics (QCD), has been extremely difficult. In the formulation of nonperturbative QCD on a Euclidean lattice, one cannot

\footnotetext{
*Corresponding author. wei.wang@sjtu.edu.cn

Corresponding author. rlzhu@njnu.edu.cn

Published by the American Physical Society under the terms of the Creative Commons Attribution 4.0 International license. Further distribution of this work must maintain attribution to the author(s) and the published article's title, journal citation, and DOI. Funded by SCOAP ${ }^{3}$.
}

directly explore time-dependent correlations. Instead, only moments of parton distribution functions, matrix elements of local operators, can be calculated. However, the difficulty in lattice QCD study grows significantly for higher moments due to technical reasons and thus only limited moments can be extracted to date [7-10].

An effective theory, called large momentum effective theory (LaMET) $[11,12]$, has been developed to compute various parton distribution functions on lattice. In this framework, an appropriate static-operator matrix element (quasiobservable) that approaches the parton observable in the infinite momentum limit of the external hadron is constructed. The quasiobservable constructed in this way is usually hadron-momentum dependent but time independent, and thus can be readily computed on the lattice. After the renormalization, the quasiobservable can be used to extract the parton observable through a factorization formula accurate up to power corrections that are suppressed by the hadron momentum. The relevant parton distribution functions calculated in the LaMET are referred as quasi-PDFs. Great progress has been made in the past few years on both the theoretical understanding of the formalism and the lattice simulations for parton distributions of baryons and mesons, see, for example, some recent reviews in Refs. [13,14].

The factorization arguments of LaMET allow us to carry out order by order perturbative calculations on the matching between the quasi-PDFs and the light-cone PDFs. This matching is one of the crucial elements in applying LaMET to parton physics. It provides a solid foundation to compute the light-cone PDFs in a systematically controlled way. In some sense, the improvement on the precision of the PDF calculations can only be achieved by combining the 
advanced lattice simulations for the quasi-PDFs (toward small lattice spacing, large volume and physical pion mass) and higher order perturbative matching calculations.

Higher order perturbative calculations are also important to demonstrate the factorization in the LaMET explicitly. In particular, some specific features of the factorization can only be manifest in the nontrivial two-loop calculations. Quasi-PDFs at one-loop order and the associated matching coefficients has been a subjective of active research since LaMET was proposed in 2013. This includes quark distribution [15-18], gluon distribution [19-21] and many others (see the review [14]). The goal of this paper is to go beyond the one-loop order and perform, for the first time, a two-loop computation of the quasi-PDF in the LaMET, taking the nondiagonal quark-quark channel as an example. This channel starts at two-loop order, which allows us to demonstrate the factorization in an intuitive method. We also notice that recently, the renormalization of quasi-PDF operators have been studied at two-loop order [22,23]. Together with this result, our paper will provide an important step toward a complete two-loop calculation of quasi-PDF and the associated matching coefficients.

The rest of this paper is organized as follows. We first present our main result of nondiagonal quark-quark splitting in LaMET at two-loop order. We will provide detailed calculations and demonstrate the factorization in detail. Based on these results, we show the matching coefficients at this order. Since the nondiagonal quark-quark splitting only starts at two-loop order, this presents the leading contribution for this channel. Some numeric results will also be presented to illustrate the behavior of the matching coefficients. We will summarize our work in the end.

\section{FACTORIZATION AT TWO-LOOP ORDER}

We start with the definitions of the light-cone PDF and quasi-PDF. For the unpolarized quark light-cone PDF, we have

$$
\begin{aligned}
f_{q / H}(x, \mu)= & \int \frac{d \xi^{-}}{4 \pi} e^{-i x p^{+} \xi^{-}}\langle p| \bar{q}\left(\xi^{-}\right) \gamma^{+} \\
& \times \exp \left(-i g \int_{0}^{\xi^{-}} d \eta^{-} A^{+}\left(\eta^{-}\right)\right) q(0)|p\rangle,
\end{aligned}
$$

where $x=k^{+} / p^{+}$is the quark longitudinal momentum fraction and $p^{\mu}=\left(p^{0}, 0,0, p^{z}\right)$ is the hadron momentum. Similarly, the quasi-PDF for the unpolarized quark is defined as

$$
\begin{aligned}
\tilde{f}_{q / H}\left(y, p^{z}\right)= & N \int \frac{d z}{4 \pi} e^{i z y p^{z}}\langle p| \bar{q}(z) \Gamma \\
& \times \exp \left(-i g \int_{0}^{z} d z^{\prime} A^{z}\left(z^{\prime}\right)\right) q(0)|p\rangle,
\end{aligned}
$$

where $z$ is a spatial direction and we will adopt $\Gamma=\gamma^{t}$ with the normalization factor $N=p^{z} / p^{t}$ and use the $\not p$ projector.
According to the factorization in the LaMET, we can write down the quasi-PDFs $\tilde{f}_{q / H}\left(y, p^{z}\right)$ in terms of the light-cone PDFs $f_{q^{\prime} / H}(x, \mu)$ :

$\tilde{f}_{q / H}\left(y, p^{z}\right)=\int_{-1}^{1} \frac{d x}{|x|}\left[C_{q q^{\prime}}\left(\frac{y}{x}, \frac{|x| p^{z}}{\mu}\right) f_{q^{\prime} / H}(x, \mu)\right]$,

with $q^{\prime}, q$ being the partons in the hadron. The $\tilde{f}_{q / H}\left(y, p^{z}\right)$ is an equal-time correlation while $f_{q^{\prime} / H}(x, \mu)$ is light-cone PDF. Though $\tilde{f}_{q / H}\left(y, p^{z}\right)$ and $f_{q^{\prime} / H}(x, \mu)$ share the same infrared structure, their ultraviolet behaviors are different, and embedded in the short-distance coefficient $C_{q q^{\prime}}$.

Since the short-distance coefficient is insensitive to the incoming hadrons, in the calculation of $C_{q q^{\prime}}$ one can replace the hadron by the partonic state. In this work we will consider the flavor nondiagonal quark contributions and the hadron state $|H\rangle$ is replaced by a quark state $\left|q^{\prime \prime}\right\rangle$ and we have the condition $q^{\prime \prime} \neq q$. We plot the Feynman diagrams for flavor nondiagonal quark distributions in Fig. 1. In our computations below, we will apply the modified minimum subtraction scheme $(\overline{\mathrm{MS}})$ and dimensional regulation with $D=4-2 \epsilon$. Under this scheme, we can write the formula for the flavor nondiagonal quark distribution as

$$
\begin{aligned}
\tilde{f}_{q / q^{\prime \prime}}\left(y, \frac{p^{z}}{\mu}, \epsilon_{\mathrm{IR}}\right) & =\int_{-1}^{1} \frac{d x}{|x|}\left[C_{q q^{\prime}}\left(\frac{y}{x}, \frac{|x| p^{z}}{\mu}\right) f_{q^{\prime} / q^{\prime \prime}}\left(x, \epsilon_{\mathrm{IR}}\right)\right] \\
& =C_{q q^{\prime}}\left(\frac{y}{x}, \frac{|x| p^{z}}{\mu}\right) \otimes f_{q^{\prime} / q^{\prime \prime}}\left(x, \epsilon_{\mathrm{IR}}\right),
\end{aligned}
$$

where both sides are computed with dimensional regulations and $\left(1 / \epsilon_{\mathrm{IR}}\right)^{n}$ represent the infrared divergences. At NNLO, the matching scheme is given as

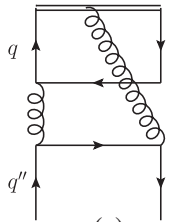

(a)

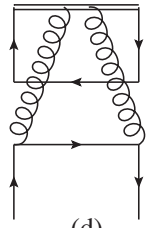

(d)

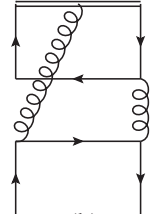

(b)

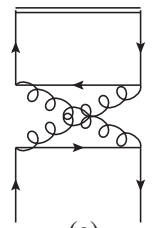

(e)

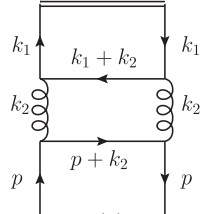

(c)

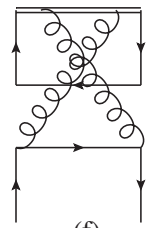

(f)
FIG. 1. Feynman diagrams for $\tilde{f}_{q / q^{\prime \prime}}$ at NNLO, where $q$ and $q^{\prime \prime}$ are quarks with different flavours. The double lines correspond to Wilson line. 


$$
\begin{aligned}
\tilde{f}_{q / q^{\prime \prime}}^{(2)}\left(y, \frac{p^{z}}{\mu}, \epsilon_{\mathrm{IR}}\right)= & C_{q q^{\prime}}^{(2)}\left(\frac{y}{x}, \frac{|x| p^{z}}{\mu}\right) \otimes f_{q^{\prime} / q^{\prime \prime}}^{(0)}\left(x, \epsilon_{\mathrm{IR}}\right) \\
& +C_{q q^{\prime}}^{(1)}\left(\frac{y}{x}, \frac{|x| p^{z}}{\mu}\right) \otimes f_{q^{\prime} / q^{\prime \prime}}^{(1)}\left(x, \epsilon_{\mathrm{IR}}\right) \\
& +C_{q q^{\prime}}^{(0)}\left(\frac{y}{x}, \frac{|x| p^{z}}{\mu}\right) \otimes f_{q^{\prime} / q^{\prime \prime}}^{(2)}\left(x, \epsilon_{\mathrm{IR}}\right) .
\end{aligned}
$$

Here, we have applied the perturbative expansions $T_{i}=$ $\sum_{n=0}^{\infty}\left(\frac{\alpha_{s}}{2 \pi}\right)^{n} T_{i}^{(n)}$ with $T_{i}$ being each of $\tilde{f}_{q / q^{\prime \prime}}, C_{q q^{\prime}}, f_{q^{\prime} / q^{\prime \prime}}$. Because of the particular feature of nondiagonal quarkquark splitting, each term at the right-hand side of the above equation represents only one contribution. In the first term, $q^{\prime}$ has to be $q^{\prime \prime}$, so that it only has $C_{q / q^{\prime \prime}}^{(2)}$. For the second term, $q^{\prime}$ has to be a gluon, and the combination is quark-to-gluon splitting $f_{g / q}^{(1)}$ and gluon-to-quark $C_{q g}^{(1)}$ matching. Finally, $q^{\prime}$ in the third term has to be $q$, representing nondiagonal quark-quark collinear splitting $f_{q / q^{\prime \prime}}^{(2)}$. We also know that both $f_{q^{\prime \prime} / q^{\prime \prime}}^{(0)}$ and $C_{q / q}^{(0)}$ are Delta functions. Therefore, the above equation can be simplified as

$$
\begin{aligned}
\tilde{f}_{q / q^{\prime \prime}}^{(2)}\left(y, \frac{p^{z}}{\mu}, \epsilon_{\mathrm{IR}}\right)= & C_{q g}^{(1)}\left(\frac{y}{x}, \frac{|x| p^{z}}{\mu}\right) \otimes f_{g / q^{\prime \prime}}^{(1)}\left(x, \epsilon_{\mathrm{IR}}\right) \\
& +C_{q q^{\prime \prime}}^{(2)}\left(y, \frac{p^{z}}{\mu}\right)+f_{q / q^{\prime \prime}}^{(2)}\left(y, \epsilon_{\mathrm{IR}}\right) .
\end{aligned}
$$

Here, $C_{q g}^{(1)}, f_{g / q^{\prime \prime}}^{(1)}$ and $f_{q / q^{\prime \prime}}^{(2)}$ are known in the literature [6,21], which are listed in the Supplemental Material [24] for reference. The objective of our calculations is to compute $\tilde{f}_{q / q^{\prime \prime}}^{(2)}$ and extract $C_{q q^{\prime \prime}}^{(2)}$. In the perturbative calculations at this order, $\tilde{f}_{q / q^{\prime \prime}}^{(2)}$ contains only IR divergences, which can be expressed as $1 / \epsilon_{\mathrm{IR}}$ in the dimensional regulation. According to the factorization theorem, the IR divergences in $\tilde{f}_{q / q^{\prime \prime}}^{(2)}$ will be canceled by that from the right-hand side of Eq. (6). In particular, the $1 / \epsilon_{\mathrm{IR}}^{2}$ term will be canceled by the last term and the $1 / \epsilon_{\mathrm{IR}}$ by the first and last term. After these cancellations, we are left with a finite term, which will be the matching coefficient at this order.

To obtain the two-loop contributions in Fig. 1, some calculation techniques are employed and we will take the subdiagram (c) as an example. In the covariant $R_{\xi}$ gauge, Fig. 1(c) contributes

$$
\begin{aligned}
\tilde{f}_{q / q^{\prime \prime}}^{(2)}\left(y, \frac{p^{z}}{\mu}\right)= & \mu^{4 \epsilon} \iint \frac{d^{4-2 \epsilon} k_{1}}{(2 \pi)^{4-2 \epsilon}} \frac{d^{4-2 \epsilon} k_{2}}{(2 \pi)^{4-2 \epsilon}} \bar{u}(p)\left(-i g T^{a} \gamma_{\mu_{2}}\right) \frac{i}{\not p+\not k_{2}}\left(-i g T^{b} \gamma_{\mu_{4}}\right) u(p) \frac{-i}{k_{2}^{2}}\left(g^{\mu_{1} \mu_{2}}-(1-\xi) \frac{k_{2}^{\mu_{1}} k_{2}^{\mu_{2}}}{k_{2}^{2}}\right) \\
& \times(-1) \operatorname{Tr}\left[\gamma^{t} \frac{i}{\not k_{1}}\left(-i g T^{b} \gamma_{\mu_{3}}\right) \frac{i}{\not k_{1}+\not k_{2}}\left(-i g T^{a} \gamma_{\mu_{1}}\right) \frac{i}{\not k_{1}}\right] \frac{-i}{k_{2}^{2}}\left(g^{\mu_{3} \mu_{4}}-(1-\xi) \frac{k_{2}^{\mu_{3}} k_{2}^{\mu_{4}}}{k_{2}^{2}}\right) \frac{\delta\left(y-\frac{k_{1}^{2}}{p^{2}}\right)}{4 N_{c} p^{t}} .
\end{aligned}
$$

In the axial gauge $A^{z}=0$, one can also easily write down the contribution by replacing the gluon propagators into $(-i) / k_{2}^{2}\left[g^{\mu_{i} \mu_{j}}-\left(n^{\mu_{i}} k_{2}^{\mu_{j}}+n^{\mu_{j}} k_{2}^{\mu_{i}}\right) /\left(n \cdot k_{2}\right)+n^{2} k_{2}^{\mu_{i}} k_{2}^{\mu_{j}} /\right.$ $\left.\left(n \cdot k_{2}\right)^{2}\right]$. Only Figs. 1(c) and 1(e) contribute in the axial gauge, where the contribution of Fig. 1(e) can be obtained from Eq. (7) by the replacement of $p \rightarrow-p$ and the replacement of gluon propagators in axial gauge.

To use the integration-by-parts technique [25] and reduce all the involved tensor integrals into a set of integrals called master integrals, we use the identity

$$
\delta\left(y-\frac{k_{1}^{z}}{p^{z}}\right)=\frac{p^{z}}{2 \pi i}\left(\frac{1}{k_{1}^{z}-y p^{z}-i 0}-\frac{1}{k_{1}^{z}-y p^{z}+i 0}\right) .
$$

The method of differential equations [26-28] is applied to calculate those master integrals. All the analytic expressions of master integrals are given in Ref. [29] by the current authors. As a specific example, all the Feynman integrals from Eq. (7) can be expressed by the first family of integrals listed in Ref. [29]. We have checked that the final results in covariant and axial gauges are consistent with each other.

As mentioned above, there is no UV divergence in flavor nondiagonal quark quasidistributions and thus it is not necessary to perform the renormalization in the modified minimal subtraction scheme. All soft divergences are also canceled. The collinear divergences in the $0<y<1$ region contain $1 / \epsilon_{\mathrm{IR}}^{2}$ and $1 / \epsilon_{\mathrm{IR}}$ :

$$
\begin{aligned}
\left.\tilde{f}_{q / q^{\prime \prime}}^{(2)}\left(y, \frac{p^{z}}{\mu}\right)\right|_{\operatorname{div}, 0<y<1}= & \frac{1}{\epsilon_{\mathrm{IR}}^{2}} \Gamma_{2}(y)+\frac{1}{\epsilon_{\mathrm{IR}}} \Gamma_{1}(y) \\
& +\frac{2}{\epsilon_{\mathrm{IR}}} \Gamma_{2}(y) \log \left(\frac{\mu^{2}}{p^{z 2}}\right),
\end{aligned}
$$

where $\Gamma_{1}$ and $\Gamma_{2}$ are defined as 


$$
\begin{aligned}
\Gamma_{1}(y)= & 2 T_{F} C_{F}\left[\frac{\left(4 y^{3}+3 y^{2}-3 y-6(y+1) y \log (y)-4\right)}{3 y} \log (2)+(y-1) \operatorname{Li}_{2}(-y)+(y+1) \operatorname{Li}_{2}(y)\right. \\
& +\frac{(y+1)\left(8 y^{2}+y-16\right) \log (y)}{12 y}+\frac{y\left(3 \pi^{2}(y-1)-y(8 y+57)+9\right)-10}{18 y}-\frac{1}{4}(3 y+7) \log ^{2}(y) \\
& \left.+\frac{(y-1)(y(4 y+7)+4) \log (1-y)}{6 y}-\frac{(y+1)(y(4 y-7)+4) \log (y+1)}{6 y}\right]
\end{aligned}
$$

$\Gamma_{2}(y)=-\frac{T_{F} C_{F}\left(4 y^{3}+3 y^{2}-3 y-6(y+1) y \log (y)-4\right)}{6 y}$.

These divergences will be canceled by two parts in Eq. (6): one from the divergences in the convolution of $C_{q g}^{(1)} \otimes f_{g q^{\prime \prime}}^{(1)}$ and the other from the divergences in $f_{q q^{\prime \prime}}^{(2)}$. Both of them are listed in the Supplemental Material [24] with known results of $C_{q g}^{(1)}$ and $f_{g / q^{\prime \prime}}^{(1)}$ at one-loop order, and $f_{q q^{\prime \prime}}^{(2)}$ at two loop. For the collinear divergences in the $-1<$ $y<0$ region, one can obtain from Eq. (9) and do the replacement $y \rightarrow-y$ and add a prefactor -1 . Note that one should do the $\log (p(y)) \rightarrow \log \left(p(y)^{2}\right) / 2$ replacement at first to avoid producing the imaginary part. The IR cancellation is similar in $y>1$ and $y<-1$ regions:

$$
\begin{aligned}
\left.\tilde{f}_{q / q^{\prime \prime}}^{(2)}\left(y, \frac{p^{z}}{\mu}, \epsilon_{\mathrm{IR}}\right)\right|_{\mathrm{div}, y>1} & =\frac{1}{\epsilon_{\mathrm{IR}}} \Gamma_{1}^{\prime}(y), \\
\left.\tilde{f}_{q / q^{\prime \prime}}^{(2)}\left(y, \frac{p^{z}}{\mu}, \epsilon_{\mathrm{IR}}\right)\right|_{\operatorname{div}, y<-1} & =-\frac{1}{\epsilon_{\mathrm{IR}}} \Gamma_{1}^{\prime}(-y),
\end{aligned}
$$

where

$$
\begin{aligned}
\Gamma_{1}^{\prime}(y)= & -\frac{T_{F} C_{F}}{3 y}\left[6(y-1) y \operatorname{Li}_{2}\left(-\frac{1}{y}\right)+22 y\right. \\
& -6(y+1) y \operatorname{Li}_{2}\left(\frac{1}{y}\right)+\left(3-4 y^{2}\right) y \log \left(\frac{y^{2}}{y^{2}-1}\right) \\
& \left.+\left(4-3 y^{2}\right) \log \left(\frac{y+1}{y-1}\right)\right] .
\end{aligned}
$$

These divergences are canceled by the convolution of $C_{q g}^{(1)} \otimes f_{g / q^{\prime \prime}}^{(1)}$ as indicated in Eq. (6). Again, we list the result in the Supplemental Material [24]. One can also see the two regions of $y>1$ and $y<-1$ are related by the symmetry of $y \rightarrow-y$ and an opposite sign.

The nontrivial cancellation of the IR divergences discussed above is an important demonstration of the LaMET factorization. This also provides a cross-check of our final result on the matching coefficient, which will be presented in the next section.

We would like to emphasize a number of points before we close this section. First, the complete cancellation of the collinear divergence depends on the factorization formula for this channel, see, Eq. (6), including the different terms contributing from the right-hand side. Second, it also depends on the exact results of lower order perturbative contributions. For example, the scale dependent term in the one-loop matching $C_{g q}^{(1)}$ (see the Supplemental Material [24]) plays a crucial role to demonstrate the complete cancellation in the above equations. This emphasizes the importance of a consistent subtraction scheme in the perturbative calculations of quasi-PDFs and the matching coefficients. Finally, our example of the nondiagonal quark-quark channel shall provide important guideline for future developments on computing the quasi-PDFs at two-loop order.

\section{MATCHING COEFFICIENT AT TWO-LOOP ORDER}

The matching coefficient $C_{q q^{\prime \prime}}^{(2)}$ is obtained by expanding both sides of Eq. (6) to $\mathcal{O}\left(\epsilon^{0}\right)$ order. Because of all the divergences between them have been canceled explicitly as shown in the previous section, it is straightforward to carry out the calculations for the finite parts.

First, let us show the result of NNLO matching coefficient $C_{q q^{\prime \prime}}^{(2)}$ in the region of $x>1$ :

$$
\begin{aligned}
\left.C_{q q^{\prime \prime}}^{(2)}\left(y, \frac{p^{z}}{\mu}\right)\right|_{y>1}= & \Gamma_{1}^{\prime}(y) \log \left(\frac{\mu^{2}}{p^{z 2}}\right)+T_{F} C_{F}\left[\left(2-\frac{8 y^{2}}{3}\right) \log ^{2}(y)-\frac{4}{9}\left(10 y^{2}+9\right) \log (y)\right. \\
& \left.-\frac{4}{3} \log (2)\left(\left(4 y^{2}-3\right) \log (y)-11\right)+\frac{4 \log ^{3}(y)}{3}-\frac{106}{9}+g_{1}(y)\right]
\end{aligned}
$$

where $\Gamma_{1}^{\prime}(y)$ has been defined in Eq. (13) and $g_{1}(y)$ is defined as 


$$
\begin{aligned}
g_{1}(y)= & 4(y+1) \operatorname{Li}_{3}\left(\frac{1}{1-y}\right)+2(y+1) \operatorname{Li}_{3}\left(\frac{1}{y}\right)-\frac{(y+1) \operatorname{Li}_{2}\left(\frac{1}{y}\right)\left(y(8 y-5)+6 y \log \left(4(y-1)^{2}\right)+8\right)}{3 y} \\
& +\frac{\left(8(y-1)(y(5 y-16)+5)-9 y(y+1) \log ^{2}\left(y^{2}\right)\right) \log \left((y-1)^{2}\right)}{36 y}+\left(\frac{4 y^{2}}{3}+y-\frac{4}{3 y}-1\right) \log (2) \log \left((y-1)^{2}\right) \\
& +\frac{\left(3 y(y+1) \log \left(y^{2}\right)+(y-1)(y(4 y+7)+4)\right) \log ^{2}\left((y-1)^{2}\right)}{12 y}-\frac{1}{12}(y+1) \log ^{3}\left((y-1)^{2}\right)+[y \rightarrow-y] .
\end{aligned}
$$

Similarly, we can obtain the matching coefficient in the $0<y<1$ region:

$$
\begin{aligned}
&\left.C_{q q^{\prime \prime}}^{(2)}\left(y, \frac{p^{z}}{\mu}\right)\right|_{0<y<1} \\
&=\Gamma_{2}(y) \log ^{2}\left(\frac{\mu^{2}}{p^{z 2}}\right)+\left(\Gamma_{1}(y)-P_{q / q^{\prime \prime}}^{(1)}(y)\right) \log \left(\frac{\mu^{2}}{p^{z 2}}\right)+T_{F} C_{F}\left[\frac{2(y-2)(y+1)^{2} \operatorname{Li}_{2}(-y)}{3 y}+\frac{1}{6}(y+13) \log ^{3}(y)\right. \\
&+\left(y^{2}+\frac{7 y}{4}+\frac{8}{3 y}+\frac{15}{4}\right) \log ^{2}(y)+\log ^{2}(2)\left(-\frac{8 y^{2}}{3}-2 y+\frac{8}{3 y}+4(y+1) \log (y)+2\right)+\frac{250 y^{2}}{27} \\
&+\frac{\pi^{2}\left(y\left(4 y^{2}-3 y+6(1-y) \log (2)-3\right)+6 y \log (y)-2\right)}{9 y}+4(y+1) \zeta(3)-\frac{419 y}{18}+\frac{56}{27 y} \\
&+\frac{\log (2)(-4 y(2 y(5 y-21)+9)+6 \log (y)(3 y(y+3)+3 y(y+3) \log (y)+8)+40)}{9 y}+\frac{1}{6} \\
&\left.+\frac{\left(-y(4 y(14 y+9)+15)+6(y-2)(y+1)^{2} \log (y+1)+40\right) \log (y)}{9 y}+g_{2}(y)\right],
\end{aligned}
$$

with $P_{q / q^{\prime \prime}}^{(1)}(y)$ being the two-loop splitting function

$$
P_{q / q^{\prime \prime}}^{(1)}(y)=\frac{T_{F} C_{F}}{2}\left[\frac{20}{9 y}-2+6 y-\frac{56 y^{2}}{9}+\left(1+5 y+\frac{8 y^{2}}{3}\right) \log (y)-(1+y) \log ^{2}(y)\right] \theta(y) \theta(1-y),
$$

and $g_{2}(y)$ is defined as

$$
\begin{aligned}
g_{2}(y)= & \operatorname{Li}_{2}(y)\left(-\frac{10 y^{2}}{3}-y-\frac{4}{y}-4(y+1) \log (2-2 y)+1\right)-4(y+1) \operatorname{Li}_{3}(1-y)-2(y+1) \operatorname{Li}_{3}(y) \\
& -\frac{(y-1)\left(3\left(y^{2}+y-2\right) \log \left(y^{2}\right)+4(y(5 y-16)+5)\right) \log (1-y)}{9 y}+\frac{2}{3} \pi^{2}(y+1) \log (1-y) \\
& +\left(1-\frac{4 y^{2}}{3}-y+\frac{4}{3 y}-2(y+1) \log (y)\right) \log ^{2}(1-y)-\frac{2(y-1)(y(4 y+7)+4) \log (2) \log (1-y)}{3 y}-[y \rightarrow-y] .
\end{aligned}
$$

In the end, the NNLO matching coefficient $C_{q q^{\prime \prime}}^{(2)}$ in $y<0$ can be obtained by replacing $y \rightarrow-y$ and adding an overall minus sign. It is interesting to investigate the asymptotic behavior of the matching coefficients at infinity points. Up to $\mathcal{O}(\epsilon)$, we have, for example,

$$
\begin{gathered}
\left.C_{q g}^{(1)}\left(y, \frac{p^{z}}{\mu}\right)\right|_{y \rightarrow+\infty}=\frac{2}{3 y} T_{F}\left[1-2 \log \left(\frac{2 y p^{z}}{\mu}\right) \epsilon\right], \\
\left.C_{q g}^{(1)}\left(y, \frac{p^{z}}{\mu}\right)\right|_{y \rightarrow-\infty}=-\frac{2}{3 y} T_{F}\left[1-2 \log \left(\frac{-2 y p^{z}}{\mu}\right) \epsilon\right] .
\end{gathered}
$$

These will lead to a logarithmic divergence when performing the integration of $C_{q g}^{(1)}\left(\frac{y}{x}, \frac{|x| p^{z}}{\mu}\right) \otimes f_{g / q^{\prime \prime}}^{(1)}\left(x, \epsilon_{\mathrm{IR}}\right)$ at infinity points. However, they do cancel between the integration at positive and negative infinity points and thus we do not need to add prescriptions to the divergence at the integration of infinity points. For the NNLO matching coefficients, we have

$$
\begin{aligned}
& \left.C_{q q^{\prime \prime}}^{(2)}\left(y, \frac{p^{z}}{\mu}\right)\right|_{y \rightarrow+\infty} \\
& \quad=\frac{4}{27 y^{2}} T_{F} C_{F}\left[-6 \log \left(\frac{\mu^{2}}{p^{z 2}}\right)+12 \log (2 y)-7\right],
\end{aligned}
$$




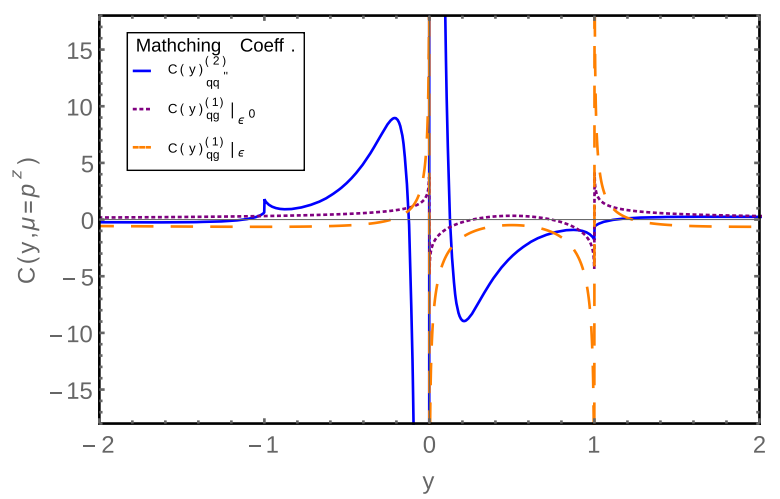

FIG. 2. Distributions of matching coefficients of $C_{q q^{\prime \prime}}^{(2)}$ and $C_{q g}^{(1)}$ as a function of momentum fraction $y$, where we adopt the scale $\mu=p^{z}$.

$$
\begin{aligned}
\left.C_{q q^{\prime \prime}}^{(2)}\left(y, \frac{p^{z}}{\mu}\right)\right|_{y \rightarrow-\infty} \\
\quad=-\frac{4}{27 y^{2}} T_{F} C_{F}\left[-6 \log \left(\frac{\mu^{2}}{p^{z 2}}\right)+12 \log (-2 y)-7\right] .
\end{aligned}
$$
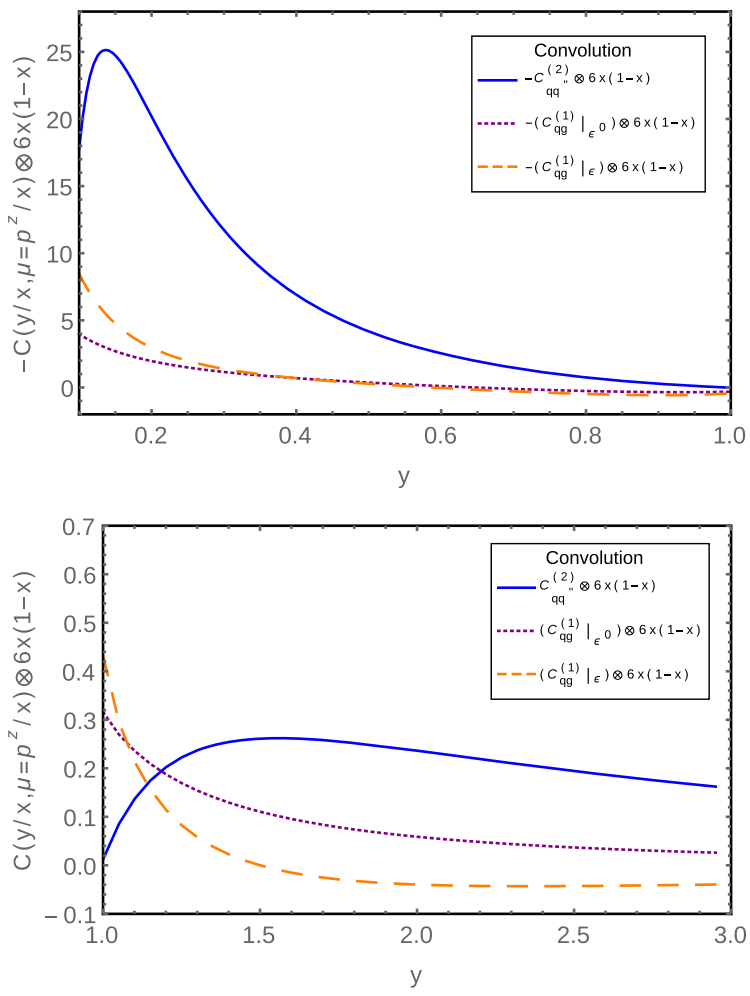

FIG. 3. Distributions of the convolution of $C_{i j}^{(n)}\left(\frac{y}{x}, \frac{p^{z}}{\mu}\right) \otimes$ $6 x(1-x)$ as a function of momentum fraction $y$, where we assume there is a toy model of $f(x)=6 x(1-x)$ with $0 \leq x \leq 1$ and adopt the scale $\mu=p^{z} / x$. Therein the top one is for $0.1<$ $y<1$ and the bottom one is for $1<y<3$.
From these, one can see $C_{q q^{\prime \prime}}^{(2)}\left(y, \frac{p^{z}}{\mu}\right)$ have better asymptotic behaviors at infinity points than $C_{q g}^{(1)}\left(y, \frac{p^{z}}{\mu}\right)$ and there will be no divergences at $\pm \infty$ if one carries out, e.g., the integral of $C_{q q^{\prime \prime}}^{(2)}\left(\frac{y}{x}, \frac{|x| p^{z}}{\mu}\right) \otimes f_{q^{\prime \prime} / q^{\prime \prime}}^{(1)}(x, \mu)$ at NNNLO matching.

We plot the distributions of matching coefficients of $C_{q q^{\prime \prime}}^{(2)}$ and $C_{q g}^{(1)}$ as a function of momentum fraction $y$ in Fig. 2. Therein the renormalization scale is adopted as $\mu=p^{z}$ and the lattice realization of $p^{z}$ is in several $\mathrm{GeV}$ currently. From it, $C_{q q^{\prime \prime}}^{(2)}$ has a different shape compared with others. Assuming the parametrization form of light-cone PDFs as the simplest one $a x^{b}(1-x)^{c}$, we can test the convolution between matching coefficients and light-cone PDFs. So we also plot the convolution of $C_{i j}^{(n)}\left(\frac{y}{x}, \frac{|x| p^{z}}{\mu}\right) \otimes 6 x(1-x)$ in Fig. 3 as a toy model. Note that $C_{q g}^{(1)}$ is divided into $\left.C_{q g}^{(1)}\left(x, \frac{p^{z}}{\mu}\right)\right|_{\epsilon^{0}}$ and $\left.C_{q g}^{(1)}\left(x, \frac{p^{2}}{\mu}\right)\right|_{\epsilon}$, where $\left.C_{q g}^{(1)}\left(y, \frac{p^{2}}{\mu}\right)\right|_{\epsilon^{0}}$ does not depend on the renormalization scale in the nonphysical region, but $\left.C_{q g}^{(1)}\left(y, \frac{p^{z}}{\mu}\right)\right|_{\epsilon}$ depends on the renormalization scale in all the region. $C_{q q^{\prime \prime}}^{(2)}$ has double logarithms as $\Gamma_{2} \log ^{2}\left(\frac{\mu^{2}}{p^{22}}\right)$ in the physical region, while single logarithms as $\Gamma_{1}^{\prime} \log \left(\frac{\mu^{2}}{p^{22}}\right)$ in the nonphysical region.

\section{CONCLUSION}

In summary, we have presented a next-to-next-to-leading order calculation of the quasiparton distribution functions for the flavor nondiagonal quark contributions $\tilde{f}_{q / q^{\prime \prime}}^{(2)}\left(y, \frac{p^{z}}{\mu}\right)$ in $-\infty<y<\infty$. We have demonstrated the LaMET factorization at this order. The matching coefficient is derived under the modified minimal subtraction scheme. These results shall be directly employed to investigate the sea quark contributions in both nonsinglet and singlet quark distributions at NNLO. This will stimulate further developments toward a complete calculation of quasi-PDFs at two-loop order and the associated matching coefficients.

\section{ACKNOWLEDGMENTS}

We thank F. Yuan for help in solving the convolution integrals, the valuable advice on our manuscript and all valuable discussions during the work. We thank X. Ji, Y.-S. Liu, J. Wang, L.-L. Yang, and Y. Zhao for valuable discussions. L. B. C. is supported by the National Natural Science Foundation of China (NSFC) under Grant No. 11805042 . W. W. is supported by NSFC under Grants No. 11735010 and No. 11911530088 , and by Natural Science Foundation of Shanghai under Grant No. 15DZ2272100. R. L. Z. is supported by NSFC under Grant No. 11705092, by Natural Science Foundation of Jiangsu under Grant No. BK20171471, by China Scholarship Council under Grant No. 201906865014 and partially supported by the U.S. Department of Energy, Office of Science, Office of Nuclear Physics, under Contract No. DE-AC02-05CH11231. 
[1] J. Gao, M. Guzzi, J. Huston, H.-L. Lai, Z. Li, P. Nadolsky, J. Pumplin, D. Stump, and C.-P. Yuan, Phys. Rev. D 89, 033009 (2014).

[2] W. Furmanski and R. Petronzio, Phys. Lett. 97B, 437 (1980).

[3] G. Curci, W. Furmanski, and R. Petronzio, Nucl. Phys. B175, 27 (1980).

[4] S. Moch, J. A. M. Vermaseren, and A. Vogt, Nucl. Phys. B646, 181 (2002).

[5] S. Moch, J. A. M. Vermaseren, and A. Vogt, Nucl. Phys. B688, 101 (2004).

[6] A. Vogt, S. Moch, and J. A. M. Vermaseren, Nucl. Phys. B691, 129 (2004).

[7] G. Martinelli and C. T. Sachrajda, Phys. Lett. B 196, 184 (1987).

[8] G. Martinelli and C. T. Sachrajda, Phys. Lett. B 217, 319 (1989).

[9] W. Detmold, W. Melnitchouk, and A. W. Thomas, Eur. Phys. J. Direct 3, 13 (2001).

[10] D. Dolgov et al. (LHPC and TXL Collaborations), Phys. Rev. D 66, 034506 (2002).

[11] X. Ji, Phys. Rev. Lett. 110, 262002 (2013).

[12] X. Ji, Sci. China Phys. Mech. Astron. 57, 1407 (2014).

[13] K. Cichy and M. Constantinou, Adv. High Energy Phys. 2019, 1 (2019).

[14] X. Ji, Y. S. Liu, Y. Liu, J. H. Zhang, and Y. Zhao, arXiv: 2004.03543.
[15] X. Xiong, X. Ji, J. H. Zhang, and Y. Zhao, Phys. Rev. D 90, 014051 (2014).

[16] T. Ishikawa, Y. Q. Ma, J. W. Qiu, and S. Yoshida, Phys. Rev. D 96, 094019 (2017).

[17] T. Izubuchi, X. Ji, L. Jin, I. W. Stewart, and Y. Zhao, Phys. Rev. D 98, 056004 (2018).

[18] X. Ji, L. C. Jin, F. Yuan, J. H. Zhang, and Y. Zhao, Phys. Rev. D 99, 114006 (2019).

[19] W. Wang, S. Zhao, and R. Zhu, Eur. Phys. J. C 78, 147 (2018).

[20] W. Wang and S. Zhao, J. High Energy Phys. 05 (2018) 142.

[21] W. Wang, J. H. Zhang, S. Zhao, and R. Zhu, Phys. Rev. D 100, 074509 (2019).

[22] X. Ji and J. H. Zhang, Phys. Rev. D 92, 034006 (2015).

[23] V. M. Braun, K. G. Chetyrkin, and B. A. Kniehl, arXiv: 2004.01043.

[24] See Supplemental Material at http://link.aps.org/ supplemental/10.1103/PhysRevD.102.011503 for the matching procedure, IR divergences and finite terms in Quasi PDFs.

[25] A. V. Smirnov, Comput. Phys. Commun. 189, 182 (2015).

[26] A. V. Kotikov, Phys. Lett. B 254, 158 (1991).

[27] A. V. Kotikov, Phys. Lett. B 267, 123 (1991); 295, 409(E) (1992).

[28] J. M. Henn, Phys. Rev. Lett. 110, 251601 (2013).

[29] L. B. Chen, W. Wang, and R. Zhu, arXiv:2006.10917. 